\title{
SYNTHESIS, SPECTRAL CHARACTERIZATION, AND ANTIPROLIFERATIVE ACTIVITY OF DISPIROPYRROLIDINES CONTAINING 2-THIOXOTHIAZOLIDIN-4-ONE NUCLEUS
}

\author{
SIVAGNANAM JAYASURIYA ${ }^{1}$, THALIGAI SUBRAMANIAN MALARVIZHI ${ }^{2}$, STEPHENKUMAR CELESTINA ${ }^{1}$, \\ KAVERI SUNDARAM ${ }^{1 *}$
}

${ }^{1}$ Departmnent of Chemistry, Karpagam Academy of Higher Education, Coimbatore, Tamil Nadu, India. ${ }^{2}$ Department of General Engineering, Muthiah Polytechnic College, Chidambaram, Tami Nadu, India. Email: sundarg2010@gmail.com

Received: 02 March 2019, Revised and Accepted: 12 April 2019

ABSTRACT

Objective: Spiro compounds are present in nature, endowed with deep biological activities. Heterocyclic compounds with a pyrrolidine scaffold are one of the paradigms of organic chemistry that exhibits a wide variety of properties and biological functions. Based on these, seven dispiropyrrolidines have been accomplished by [3+2] cycloaddition reaction from acenaphthenequinone and sarcosine with several dipolaro files such as substituted 5-benzylidene-2-thioxothiazolidin-4-ones.

Methods: Cycloadducts 4a-g were prepared by conventional method and the structures of the compounds $\mathbf{4 a - g}$ were completely characterized by infrared, ${ }^{1} \mathrm{H},{ }^{13} \mathrm{C}$ nuclear magnetic resonance spectral data, and elemental analysis. The cytotoxic activity of the synthesized compounds was carried out by 3-(4,5-dimethylthiazol-2-yl)-2,5-diphenyl tetrazolium bromide assay.

Results: The dispiropyrrolidines $\mathbf{4 a - g}$ were showed a moderate-to-good cytotoxic activity against human cervical cancer lines. Among all the synthesized compounds, $\mathbf{4 d}$ was found to be more potent with human cervical cancer line with an half maximal inhibitory concentration $\left(\mathrm{IC}_{50}\right.$ ) value of $5.5 \mu \mathrm{M}$.

Conclusion: The synthesized compound $\mathbf{4 d}$ found to be an excellent activity which is nearly closed to reference drug gemcitabine with an IC ${ }_{50}$ value of $4.6 \mu \mathrm{M}$.

Keywords: Rhodanine, Acenaphthenequinone, Sarcosine, Antiproliferative activity.

(C) 2019 The Authors. Published by Innovare Academic Sciences Pvt Ltd. This is an open access article under the CC BY license (http://creativecommons. org/licenses/by/4. 0/) DOI: http://dx.doi.org/10.22159/ajpcr.2019.v12i5.32902

\section{INTRODUCTION}

The 1,3-dipolar cycloaddition reactions [1] have been established as an efficient method for the construction of five-membered nitrogen heterocycles. These five-member heterocycles are very important due to their high regioselectivity and stereoselectivity. A pyrrolidine scaffold is one of the paradigms of organic chemistry that exhibits a wide variety of properties and biological functions [2]. Spirooxindole cores are attractive synthetic targets due to their prevalence in numerous natural products and applications in medicine and therapeutics [3]. In addition, the spirooxindole ring systems are the central skeleton of numerous alkaloids and therapeutically important compounds. More serious diseases such as cancer are considered the second leading cause of death worldwide after cardiovascular diseases. Only in the USA as of $2015,589,430$ cancer patients died and more than 1,658,370 million new cancer cases were identified according to the 2015 cancer data and figures report and it will be an estimated 18.1 million new cancer cases and 9.6 million cancer deaths in 2018 [4]. More than 13 million deaths are expected to occur worldwide by 2030 . In India, approximately 1 million new cases occur each year. This is $15 \%$ less than in the United States, whose population is one-third that of India. The disturbing fact is that the number of India is expected to double in 20 years, according to the International Agency for Research on Cancer. Cervical cancer is dynamically associated with infection by oncogenic types of human papillomavirus (HPV), which is critical cancer in women. There are more than 100 types of HPV, many of which infect the genital tract. Almost $80 \%$ of cases occur in low-income countries or developing countries. This emphasizes that the incessant need to develop new classes of anticancer agents is an important and challenging goal in medical chemistry. Rhodanine-based analogs have been associated with several biological activities such as antibacterial [5,6], antifungal [7], antiviral [8], antimalarial [9], insecticide [10], anti-inflammatory [11], and antidiabetic [12]. Rhodanine and its related compounds are probably due to its affinity for the anticancer agents such as JNKstimulating phosphate-1 (JSP-1) [13], tumor necrosis factor [14], and antiapoptotic biocomplex BCLXL BH3 [15]. In addition, rhodanine and its analogs have wide industrial applications as vulcanizing agents of synthetic rubber, extreme pressure lubricants, intermediates in the synthesis of dyes, antioxidants, and polishing additives in silver electroplating [16]. The present study has been reported through a highly selective regio- and stereo-scopic cycloaddition reaction derived from acenaphthenequinone and sarcosine with several dipolaro files such as substituted 5-benzylidene-2-thioxothiazolidin-4-ones.

\section{METHODS}

\section{Experiments}

The complete chemicals were used as laboratory grade purchased from HiMedia Company. The melting points were determined in a digital melting point instrument XT-5 and are incorrect. The infrared (IR) spectra were recorded on a Shimadzu 360 FT-IR spectrometer. The ${ }^{1} \mathrm{H}$ nuclear magnetic resonance (NMR) and ${ }^{13} \mathrm{C}$ NMR spectra were measured at 400 and $125 \mathrm{MHz}$, respectively, on a Bruker-400 spectrometer using TMS as internal standard and DMSO- $\mathrm{d}_{6}$ as a solvent. The elemental analyses for $\mathrm{C}, \mathrm{H}, \mathrm{N}$, and $\mathrm{S}$ were $\pm 0.04 \%$ of the theoretical values and determined using a PerkinElmer 240C elemental analyzer.

General procedure for the synthesis of dispiropyrrolidine derivatives (4a-g)

A mixture of acenaphthenequinone $(0.0015 \mathrm{~mol})$, sarcosine $(0.0015 \mathrm{~mol})$, and substituted 5-benzylidene-2-thioxothiazolidin-4-ones $(0.0015 \mathrm{~mol})$ was dissolved in methanol-1,4-dioxane and refluxed for 15-18 h. After 
completing the reaction as evident by thin-layer chromatography, the mixture was poured into ice water. The precipitated solid was filtered and purified by recrystallization using ethanol.

1 - $N$ - methyl-spiro $\left[2.3^{1}\right]$ a cenaphthene- $4^{1}$ - (phenyl)spiro $\left[3.5^{1}\right] 2^{11}, 4^{11}$-thiazolidin-one-3,3 $3^{1}$ pyrrolidine (4a)

${ }^{1} \mathrm{H}$ NMR: $\delta 2.028$ (H-9), 3.648 (H-7a), 3.916 (H-7b), 4.594 (H-6), 7. 432 (H-4'), $7.532\left(\mathrm{H}-5^{\prime \prime}, 9^{\prime \prime}, 10^{\prime \prime}\right), 7.678\left(\mathrm{H}-2^{\prime}, 6^{\prime}\right), 7.874\left(\mathrm{H}-3^{\prime}, 5^{\prime}\right), 7.952(\mathrm{H}-$ $\left.8^{\prime \prime}\right), 8.136\left(\mathrm{H}-4^{\prime \prime}\right), 8.349\left(\mathrm{H}-6^{\prime \prime}\right), 13.242(\mathrm{H}-3) \cdot{ }^{13} \mathrm{C}$ NMR: $\delta 34.876(\mathrm{C}-$ 9), 49.03 (C-6), 52.09 (C-7), 77.48 (C-5), 81.64 (C-1"), 121.69 (C-5"), 122.32 (C-4'), 123. $96\left(\mathrm{C}-10^{\prime \prime}\right), 127.18\left(\mathrm{C}-8^{\prime \prime}\right), 129.48\left(\mathrm{C}-3^{\prime}, 5^{\prime}, 9^{\prime \prime}\right)$, 130.58 (C-4"), 130. $81\left(\mathrm{C}-3^{\prime \prime}\right), 132.15\left(\mathrm{C}-2^{\prime}, 6^{\prime}\right), 132.54\left(\mathrm{C}-4^{\prime}\right), 133.06$ (C-11"), 133.42 (C-6"), 133.59 (C-7"), 137.314 (C-1'), 143.48 (C-12"), 180.07 (C-4), 201.23 (C-2"), 198.40(C-2). Anal. Calc. for $\mathrm{C}_{24} \mathrm{H}_{18} \mathrm{~N}_{2} \mathrm{O}_{2} \mathrm{~S}$ : C, $66.95 ; \mathrm{H}, 4.21$; N, 6.51; 0, 7.43; S, 14.90. Found: C, 66.97; H, 4.20; N, 6.54; $0,7.46 ;$ S, 14.87 .

1 - $N$-methyl-spiro[2.3 $]$ acenaphthene- $4^{1}$-(4-bromophenyl)-

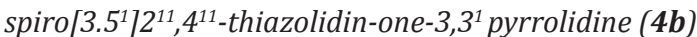

${ }^{1} \mathrm{H}$ NMR: $\delta 2.021$ (H-9), 3.658 (H-7a), 3.926 (H-7b), 4.586 (H-6), 7.542 $\left(\mathrm{H}-5^{\prime \prime}, 9^{\prime \prime}, 10^{\prime \prime}\right), 7.652\left(\mathrm{H}-2^{\prime}, 6^{\prime}\right), 7.876\left(\mathrm{H}-3^{\prime}, 5^{\prime}\right), 7.948\left(\mathrm{H}-8^{\prime \prime}\right), 8.146(\mathrm{H}-$ $\left.4^{\prime \prime}\right), 8.352\left(\mathrm{H}-6^{\prime \prime}\right), 13.238(\mathrm{H}-3){ }^{13} \mathrm{C}$ NMR: $\delta 34.868$ (C-9), $49.04(\mathrm{C}-6)$, 52.02 (C-7), 77.38 (C-5), 81.68 (C-1"), 121.78 (C-5"), 123.98 (C-10"), $127.11\left(\mathrm{C}-8^{\prime \prime}\right), 129.38\left(\mathrm{C}-3^{\prime}, 5^{\prime}, 9^{\prime \prime}\right), 130.69\left(\mathrm{C}-4^{\prime \prime}\right), 130.86\left(\mathrm{C}-3^{\prime \prime}\right)$, $132.14\left(\mathrm{C}-2^{\prime}, 6^{\prime}\right), 132.56\left(\mathrm{C}-4^{\prime}\right), 133.08\left(\mathrm{C}-11^{\prime \prime}\right), 133.39$ (C-6"), 133.54 (C-7"), 137.311 (C-1'), 143.48 (C-12"), 179.09 (C-4), 206.91 (C-2"), 199.56 (C-2). Anal. Calc. for $\mathrm{C}_{24} \mathrm{H}_{17} \mathrm{BrN}_{2} \mathrm{O}_{2} \mathrm{~S}_{2}: \mathrm{C}, 56.58 ; \mathrm{H}, 3.36 ; \mathrm{Br}, 15.68$; N, 5.50; 0, 6.28; S, 12.59. Found: C, 56.60; H, 3.38; Br, 15.65; N, 5.53; O, $6.31 ; \mathrm{S}, 12.57$.

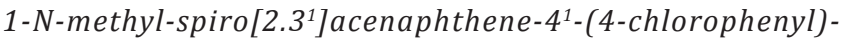
spiro $\left[3.5^{1}\right] 2^{11}, 4^{11}$-thiazolidin-one-3,31 $3^{1}$ pyrrolidine $(\mathbf{4 c})$

${ }^{1} \mathrm{H}$ NMR: $\delta 2.025$ (H-9), 3.644 (H-7a), 3.919 (H-7b), 4.590 (H-6), 7.534 $\left(\mathrm{H}-5^{\prime \prime}, 9^{\prime \prime}, 10^{\prime \prime}\right), 7.675\left(\mathrm{H}-2^{\prime}, 6^{\prime}\right), 7.870\left(\mathrm{H}-3^{\prime}, 5^{\prime}\right), 7.956\left(\mathrm{H}-8^{\prime \prime}\right), 8.139(\mathrm{H}-$ $\left.4^{\prime \prime}\right), 8.348\left(\mathrm{H}-6^{\prime \prime}\right), 13.241$ (H-3). ${ }^{13} \mathrm{C}$ NMR: $\delta 34.877$ (C-9), 49.06 (C-6), 52.04 (C-7), 77.32 (C-5), 81.66 (C-1"), $121.75\left(\mathrm{C}-5^{\prime \prime}\right), 123.94$ (C-10"), 127.14 (C-8"), 129. $31\left(\mathrm{C}-3^{\prime}, 5^{\prime}, 9^{\prime \prime}\right), 130.60\left(\mathrm{C}-4^{\prime \prime}\right), 130.83\left(\mathrm{C}-3^{\prime \prime}\right)$, 132.17 (C-2',6'), 132. 51 (C-4'), 133.03 (C-11"), 133.35 (C-6"), 133.59 (C-7"), 137.311 (C-1'), 143.45 (C-12"), 179.23(C-4), 206.81(C-2"), 199.67(C-2). Anal. Calc. for $\mathrm{C}_{24} \mathrm{H}_{17} \mathrm{ClN}_{2} \mathrm{O}_{2} \mathrm{~S}_{2}: \mathrm{C}, 61.99 ; \mathrm{H}, 3.69 ; \mathrm{Cl}, 7.62$; N, 6.02; 0, 6.88; S, 13.79. Found: C, 61.96; H, 3.65; Cl, 7.65; N, 6.06; O, 6.90; S, 13.81 .

1-N-methyl-spiro $\left[2.3^{1}\right]$ acenaphthene-4 $4^{1}$ (4-fluorophenyl)spiro $\left[3.5^{1}\right] 2^{11}, 4^{11}$-thiazolidin-one-3,3 $3^{1}$ pyrrolidine $(\mathbf{4 d})$

${ }^{1} \mathrm{H}$ NMR: $\delta 2.032$ (H-9), 3.638 (H-7a), 3.914 (H-7b), 4.598 (H-6), 7.538 $\left(\mathrm{H}-5^{\prime \prime}, 9^{\prime \prime}, 10^{\prime \prime}\right), 7.665\left(\mathrm{H}-2^{\prime}, 6^{\prime}\right), 7.868\left(\mathrm{H}-3^{\prime}, 5^{\prime}\right), 7.952\left(\mathrm{H}-8^{\prime \prime}\right), 8.132(\mathrm{H}-$ $\left.4^{\prime \prime}\right), 8.339\left(\mathrm{H}-6^{\prime \prime}\right), 13.246(\mathrm{H}-3){ }^{13} \mathrm{C}$ NMR: $\delta 34.869$ (C-9), 49.05 (C-6), 52.02 (C-7), 77.36 (C-5), 81.68 (C-1"), 121.79 (C-5"), 123. 92 (C-10"), $127.17\left(\mathrm{C}-8^{\prime \prime}\right), 129.35\left(\mathrm{C}-3^{\prime}, 5^{\prime}, 9^{\prime \prime}\right), 130.63\left(\mathrm{C}-4^{\prime \prime}\right), 130.87\left(\mathrm{C}-3^{\prime \prime}\right), 132.16$ (C-2',6'), 132. 55 (C-4'), $133.06\left(\mathrm{C}-11^{\prime \prime}\right), 133.39$ (C-6"), 133.52 (C-7"), $137.316\left(\mathrm{C}-1^{\prime}\right), 143.48\left(\mathrm{C}-12^{\prime \prime}\right), 178.22$ (C-4), 203.61(C-2"), 199.52 (C2). Anal. Calc. for $\mathrm{C}_{24} \mathrm{H}_{17} \mathrm{FN}_{2} \mathrm{O}_{2} \mathrm{~S}_{2}: \mathrm{C}, 64.27 ; \mathrm{H}, 3.82 ; \mathrm{F}, 4.24 ; \mathrm{N}, 6.25 ; 0,7.13$; S, 14.30. Found: C, 64.30; H, 3.83; F, 4.20; N, 6.21; 0, 7.15; S, 14.32 .

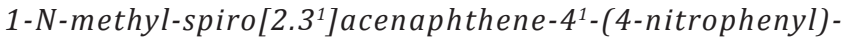
spiro $\left[3.5^{1}\right] 2^{11}, 4^{11}$-thiazolidin-one-3,3 $3^{1}$ pyrrolidine (4e)

${ }^{1} \mathrm{H}$ NMR: $\delta 2.04$ (H-9), 3.698 (H-7a), 3.70 (H-7b), 4.75 (H-6), 7.672 (H$\left.5^{\prime \prime}, 9^{\prime \prime}, 10^{\prime \prime}\right), 7.731\left(\mathrm{H}-2^{\prime}, 6^{\prime}\right), 7.869\left(\mathrm{H}-3^{\prime}, 5^{\prime}\right), 7.951\left(\mathrm{H}-8^{\prime \prime}\right), 8.139$ (H-4"), $8.372\left(\mathrm{H}-6^{\prime \prime}\right), 13.26(\mathrm{H}-3) .{ }^{13} \mathrm{C}$ NMR: $\delta 34.89$ (C-9), 49.12 (C-6), 58.61 (C7), 76.70 (C-5), 81.72 (C-1"), 121.85 (C-5"), 123.62 (C-10"), 127.34 (C$\left.8^{\prime \prime}\right), 129.23\left(\mathrm{C}-3^{\prime}, 5^{\prime}, 9^{\prime \prime}\right), 130.64\left(\mathrm{C}-4^{\prime \prime}\right), 130.92\left(\mathrm{C}-3^{\prime \prime}\right), 132.19\left(\mathrm{C}-2^{\prime}, 6^{\prime}\right)$, 132. 54 (C-4'), 133.08 (C-11"), 133.39 (C-6"), 133.39 (C-7"), 137.326 (C-1'), 147.54 (C-12"), 178.94 (C-4), 206.84(C-2"), 199.25(C-2). Anal. Calc. for $\mathrm{C}_{24} \mathrm{H}_{17} \mathrm{~N}_{3} \mathrm{O}_{4} \mathrm{~S}_{2}$ : C, 60.62; $\mathrm{H}, 3.60 ; \mathrm{N}, 8.84 ; 0,13.46 ; \mathrm{S}, 13.49$. Found: C, 60.59; H, 3.61; N, 8.80; O, 13.49; S, 13.47 .
1-N-methyl-spiro $\left[2.3^{1}\right]$ acenaphthene-4 ${ }^{1}$-(4-methylphenyl)spiro[3.5 $] 2^{11}, 4^{11}$-thiazolidin-one-3,3 $3^{1}$ pyrrolidine $(\mathbf{4 f})$

${ }^{1} \mathrm{H}$ NMR: $\delta 2.036$ (H-9), 2.228 (H-1"'), 3.642 (H-7a), 3.921 (H-7b), 4.594 (H-6), $7.542\left(\mathrm{H}-5^{\prime \prime}, 9^{\prime \prime}, 10^{\prime \prime}\right), 7.673\left(\mathrm{H}-2^{\prime}, 6^{\prime}\right), 7.862\left(\mathrm{H}-3^{\prime}, 5^{\prime}\right), 7.955(\mathrm{H}-$ $\left.8^{\prime \prime}\right), 8.135\left(\mathrm{H}-4^{\prime \prime}\right), 8.342\left(\mathrm{H}-6^{\prime \prime}\right), 13.243(\mathrm{H}-3){ }^{13} \mathrm{C}$ NMR: $\delta 24.39$ (C1'") 34.872 (C-9), 49.03 (C-6), 52.11 (C-7), 77.42 (C-5), 81.63 (C-1"), $121.81\left(\mathrm{C}-5^{\prime \prime}\right), 123.95\left(\mathrm{C}-10^{\prime \prime}\right), 127.12\left(\mathrm{C}-8^{\prime \prime}\right), 129.42\left(\mathrm{C}-3^{\prime}, 5^{\prime}, 9^{\prime \prime}\right)$, 130.71 (C-4"), 130. 89 (C-3"), 132.09 (C-2',6'), 132. 43 (C-4'), 133.08 (C-11"), 133.45 (C-6"), 133.42 (C-7"), $137.319\left(\mathrm{C}-1^{\prime}\right), 135.24\left(\mathrm{C}-4^{\prime}\right)$ 143.52 (C-12"), 178.99 (C-4), 206.02 (C-2"), 199.32 (C-2). Anal. Calc. for $\mathrm{C}_{25} \mathrm{H}_{20} \mathrm{~N}_{2} \mathrm{O}_{2} \mathrm{~S}_{2}$ : C, 67.54; H, 4.53; N, 6.30; O, 7.20; S, 14.43; Found: C, $67.58 ; \mathrm{H}, 4.54 ; \mathrm{N}, 6.32 ; 0,7.18 ; \mathrm{S}, 14.46$

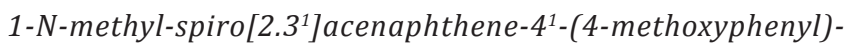
spiro[3.5 $\left.{ }^{1}\right] 2^{11}, 4^{11}$-thiazolidin-one-3, $3^{1}$ pyrrolidine $(\mathbf{4 g})$

${ }^{1} \mathrm{H}$ NMR: $\delta 2.038$ (H-9), 3.98 (H-1"'), 3.652 (H-7a), 3.941 (H-7b), 4.586 $(\mathrm{H}-6), 7.548\left(\mathrm{H}-5^{\prime \prime}, 9^{\prime \prime}, 10^{\prime \prime}\right), 7.675\left(\mathrm{H}-2^{\prime}, 6^{\prime}\right), 7.869\left(\mathrm{H}-3^{\prime}, 5^{\prime}\right), 7.952(\mathrm{H}-$ $\left.8^{\prime \prime}\right), 8.138\left(\mathrm{H}-4^{\prime \prime}\right), 8.345\left(\mathrm{H}-6^{\prime \prime}\right), 13.246(\mathrm{H}-3){ }^{13} \mathrm{C}$ NMR: $\delta 34.876(\mathrm{C}-$ 9), 49.12 (C-6), 52.19 (C-7), $55.65\left(\mathrm{C}-1^{\prime \prime \prime}\right) 77.49$ (C-5), $81.75\left(\mathrm{C}-1^{\prime \prime}\right)$, $121.84\left(\mathrm{C}-5^{\prime \prime}\right), 123.97\left(\mathrm{C}-10^{\prime \prime}\right), 127.14\left(\mathrm{C}-8^{\prime \prime}\right), 129.51\left(\mathrm{C}-3^{\prime}, 5^{\prime}, 9^{\prime \prime}\right)$, $130.75\left(\mathrm{C}-4^{\prime \prime}\right), 130.92\left(\mathrm{C}-3^{\prime \prime}\right), 132.19\left(\mathrm{C}-2^{\prime}, 6^{\prime}\right), 132.44\left(\mathrm{C}-4^{\prime}\right), 133.12$ (C-11"), 133.36 (C-6"), 133.51 (C-7"), 137.321 (C-1'), 143.51 (C-12"), 159.02 (C-4'), 179.99 (C-4), 204.52 (C-2"), 199.01 (C-2). Anal. Calc. for $\mathrm{C}_{25} \mathrm{H}_{20} \mathrm{~N}_{2} \mathrm{O}_{3} \mathrm{~S}_{2}$ : C, 65.20; H, 4.38; N, 6.08; 0, 10.42; S, 13.92; Found: C, $65.23 ; \mathrm{H}, 4.34 ; \mathrm{N}, 6.10 ; 0,10.45 ; \mathrm{S}, 13.90$.

3-(4,5-dimethylthiazol-2-yl)-2,5-diphenyl tetrazolium bromide (MTT) assay for cytotoxicity screening

The human cervical cancer cell line was obtained from the National Centre for Cell Science (NCCS), Pune, and cultured in Eagle's minimal essential medium containing 10\% fetal bovine serum (FBS). The cells were maintained at $37^{\circ} \mathrm{C}, 5 \% \mathrm{CO}_{2}, 95 \%$ air, and $100 \%$ relative humidity. Extracts were added to cells cultured in concentrations of $6.25,12.5,25$, 50 , and $100 \mu \mathrm{g}$ of stock of $1 \mathrm{mg} / \mathrm{ml} \mathrm{0.1 \%} \mathrm{DMSO} \mathrm{and} \mathrm{incubated} \mathrm{for} 24 \mathrm{~h}$. The percentage difference in viability was determined by a standard MTT assay after $48 \mathrm{~h}$ of incubation. The percentage of cell viability was then calculated with respect to control as follows:

$\%$ Cell viability $=[\mathrm{A}]$ Test $/[\mathrm{A}]$ control $\times 100$

\section{RESULTS AND DISCUSSION}

\section{Chemistry}

5-benzylidene-2-thioxothiazolidin-4-ones were prepared from rhodanine and various substituted aromatic aldehydes according to the method previously reported [17]. Cycloadducts 4a-g were prepared by the reaction of different substituted 5-benzylidene-2thioxothiazolidin-4-ones 3a-g with azomethine ylide [18] obtained from acenaphthenequinone $\mathbf{1}$ and sarcosine $\mathbf{2}$ (Scheme 1).

The formation of dispiropyrrolidines $\mathbf{4 a - g}$ was confirmed by spectral $\left(\mathrm{IR},{ }^{1} \mathrm{H} \mathrm{NMR}\right.$, and $\left.{ }^{13} \mathrm{C} \mathrm{NMR}\right)$ and elemental analysis. Among the synthesized compounds, 4c (Fig. 1) was taken as a representative compound for spectroscopic discussion. The IR spectrum of adduct $\mathbf{4 c}$ showed carbonyl at $1730 \mathrm{~cm}^{-1}$ and thiocarbonyl resonated at $1200 \mathrm{~cm}^{-1}$.

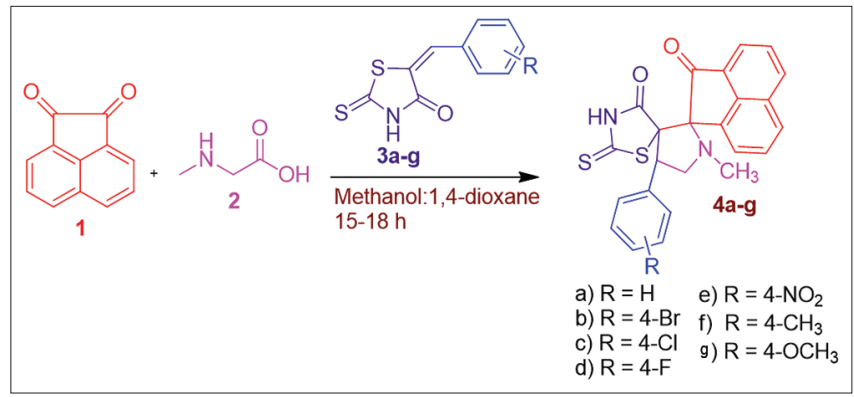

Scheme 1: Synthesis of titled compounds 4a-g 


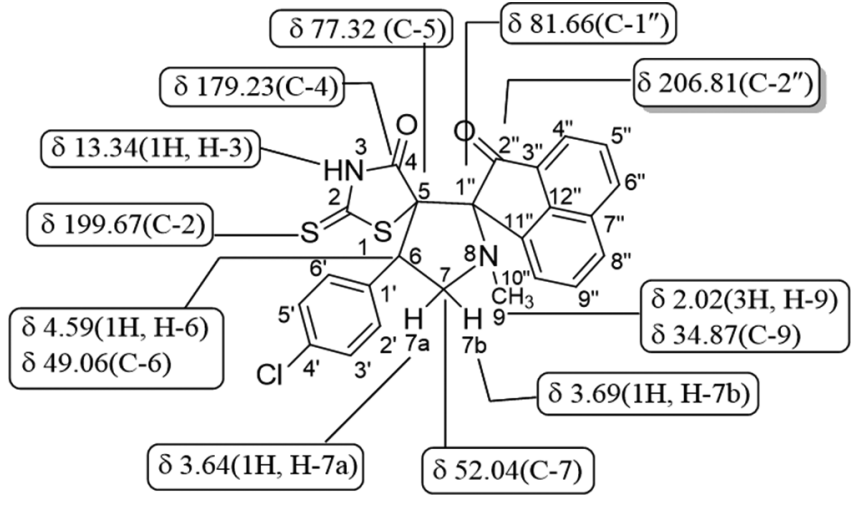

Fig. 1: Selected ${ }^{1} \mathrm{H}$ and ${ }^{13} \mathrm{C}$ chemical shifts of compound $4 \mathrm{c}$

Table 1: In vitro antiproliferative activity of compounds $4 \mathrm{a}-\mathrm{g}$ by MTT assay

\begin{tabular}{ll}
\hline Compound & $\mathbf{I C}_{50}( \pm \mathbf{S D}) \boldsymbol{\mu M}$ \\
\hline $4 \mathrm{a}$ & $36.8( \pm 1.12)$ \\
$4 \mathrm{~b}$ & $17.4( \pm 0.04)$ \\
$4 \mathrm{c}$ & $24.2( \pm 0.12)$ \\
4d & $05.8( \pm 0.46)$ \\
$4 \mathrm{e}$ & $14.5( \pm 0.72)$ \\
$4 \mathrm{f}$ & $25.4( \pm 0.35)$ \\
4g & $28.6( \pm 0.94)$ \\
Gemcitabine & $04.6( \pm 0.44)$ \\
\hline IC
\end{tabular}

$\mathrm{IC}_{50}$ values represent the concentration required to decrease cytotoxic activity by $50 \%$. IC $\mathrm{C}_{5}$ : Half maximal inhibitory concentration, SD: Standard deviation, MTT: 3-(4,5-dimethylthiazol-2-yl)-2,5-diphenyl tetrazolium bromide

The sharp peak appeared at $2992 \mathrm{~cm}^{-1}$ for the NH group of rhodanine moiety.

In the ${ }^{1} \mathrm{H}$ NMR spectrum (Fig. 1) of compound $\mathbf{4 c}$, the sharp singlet at $\delta$ 2.02 indicates $\mathrm{N}-\mathrm{CH}_{3}$ protons $(\mathrm{H}-9)$. The pyrrolidine ring $\mathrm{N}-\mathrm{CH}_{2}$ protons $(\mathrm{H}-7 \mathrm{a}$ and $7 \mathrm{~b})$ appeared as a doublet of doublet at $\delta 3.64$ and 3.69. The benzylic proton (H-6) appeared as a doublet at $\delta 4.59$ and the product 4c exhibited a multiplet at a range $\delta$ 7.53-8.34 due to aromatic protons. The peak appeared at $\delta 13.24$ is due to $\mathrm{NH}$ proton $(\mathrm{H}-3)$ of rhodanine moiety. The cycloadduct $4 \mathrm{c}$ also confirmed by its ${ }^{13} \mathrm{C}$ NMR spectrum (Fig. 1), signals at $\delta 77.32$ and $\delta 81.66$ due to two spiro carbons C-5 and $\mathrm{C}-1^{\prime \prime}$, respectively. Pyrrolidine ring $\mathrm{N}-\mathrm{CH}_{3}$ carbon C-9 resonated at $\delta 34.87$ and $\mathrm{N}-\mathrm{CH}_{2}$ carbon C-7 also observed at $\delta 52.04 \mathrm{ppm}$. The signal at $\delta 49.06$ ppm due to benzylic carbon C- 6 and the bunch of peaks at $\delta$ 121.75 to $\delta 143.45 \mathrm{ppm}$ indicates aromatic carbons. The two carbonyl groups observed at $\delta 206.81\left(\mathrm{C}-2^{\prime \prime}\right)$ and $\delta 179.23(\mathrm{C}-4) \mathrm{ppm}$ and the thiocarbonyl group appeared at $\delta 199.67(\mathrm{C}-2)$. All other cycloadducts were exhibited identical results.

\section{Antiproliferative activity}

In vitro antiproliferative activity for synthesized compounds, 4a-g was evaluated by the measurement of their cytotoxic properties against human cervical cancer by MTT assay [19] and their results were expressed as half maximal inhibitory concentration $\left(\mathrm{IC}_{50}\right)$ and depicted in Table 1. In addition, gemcitabine was used as a standard drug.

As shown from Table 1, the synthesized compounds showed moderateto-good antiproliferative activity against human cervical cancer cell line with the $\mathrm{IC}_{50}$ values from $5.8 \mu \mathrm{M}$ to $36.8 \mu \mathrm{M}$. Among the seven tested compounds, compound $\mathbf{4 d}$ showed a better antiproliferative activity due to the higher electronegativity of fluorine substitution. Further, compound $\mathbf{4 e}$ carried nitro atom on the phenyl ring was found to be more potency with the $\mathrm{IC}_{50}$ value of $14.5 \mu \mathrm{M}$. For bromo and chloro substituent, $\mathbf{4 b}$ and $\mathbf{4 c}$ exhibited an improved antiproliferative activity with the $\mathrm{IC}_{50}$ values $17.4 \mu \mathrm{M}$ and $24.2 \mu \mathrm{M}$, respectively. On the other hand, the electron donating substituents $\mathbf{4 f}$ and $\mathbf{4 g}$ found to be moderate potential against human cervical cancer cell lines ( $\mathrm{IC}_{50} 25.4$ $\mu \mathrm{M}$ and $28.6 \mu \mathrm{M}$, respectively). However, the unsubstituted compound 4a displayed a lower activity compared to all other synthesized compounds. Among the tested compounds, compound $\mathbf{4 d}$ found to be an excellent activity which is nearly closed to reference drug gemcitabine ( $\left.\mathrm{IC}_{50} 4.6 \mu \mathrm{M}\right)$.

\section{CONCLUSION}

We have synthesized seven cycloadducts from acenaphthenequinone and sarcosine with several dipolaro files such as substituted 5-benzylidene-2-thioxothiazolidin-4-ones and examined their antiproliferative activity against human cervical cancer cell lines by MTT assay. It seems that the electron withdrawing substituents $\mathbf{4 b}, \mathbf{4 c}, \mathbf{4 d}$, and $\mathbf{4 e}$ enhanced the activity with $\mathrm{IC}_{50}$ values $17.4,24.2$, 5.8 , and $14.5 \mu \mathrm{M}$, respectively, and more dominant than electron donating substituents such as $4 \mathrm{f}$ and $4 \mathrm{~g}$ with the $\mathrm{IC}_{50}$ values are 25.4 and $28.6 \mu \mathrm{M}$. Among the tested compounds, compound $\mathbf{4 d}$ showed an excellent activity than all other compounds. As from the results, we may conclude that the electron withdrawing substituents showed better antiproliferative activity than electron donating substituents.

\section{ACKNOWLEDGMENTS}

The authors wish to express their gratitude to the Karpagam Academy of Higher Education (Deemed to be University) for providing infrastructure specialties.

\section{AUTHORS' CONTRIBUTION}

All authors have an equal contribution for this article.

\section{CONFLICTS OF INTEREST}

The authors declare that we have no conflicts of interest.

\section{REFERENCES}

1. Padwa A. In: 1,3-Dipolar Cycloaddition Chemistry. Vol. 1 and 2. New York: Wiley; 1984

2. Raj AA, Raghunathan R. A novel entry into a new class of spiroheterocyclic framework: Regioselective synthesis of dispiro [oxindole-cyclohexanone] pyrrolidines and dispiro [oxindolehexahydroindazole] pyrrolidines. Tetrahedron 2001;57:10293-8.

3. Galliford CV, Scheidt KA. Pyrrolidinyl-spirooxindole natural products as inspirations for the development of potential therapeutic agents. Angew Chem Int Ed Engl 2007;46:8748-58.

4. Bray F, Ferlay J, Soerjomataram I, Siegel RL, Torre LA, Jemal A, et al. Global cancer statistics 2018: GLOBOCAN estimates of incidence and mortality worldwide for 36 cancers in 185 countries. CA Cancer J Clin 2018;68:394-424.

5. Song MX, Zheng CJ, Deng XQ, Wang Q, Hou SP, Liu TT, et al. Synthesis and bioactivity evaluation of rhodanine derivatives as potential anti-bacterial agents. Eur J Med Chem 2012;54:403-12.

6. Pooja P, Meenakshi D, Amol K, Ganesh N, Smita P. Design, synthesis, docking studies and biological evaluation of 2-phenyl-3-(substituted benzo[d] thiazol 2-ylamino)-quinazoline-4(3h)-one derivatives as antimicrobial agents. Int J Pharm Pharm Sci 2018;10:57-61.

7. Khan FA, Sangshetti JN. Design, synthesis and molecular docking study of hybrid quinoline-4-yloxadiazoles/oxathiadiazoles as potent antifungal agents. Int J Pharm Pharm Sci 2015;7:222-9.

8. Wu JH, Wang XH, Yi YH, Lee KH. Anti-AIDS agents 54. A potent anti-HIV chalcone and flavonoids from genus desmos. Bioorg Med Chem Lett 2003;13:1813-5.

9. Kumar G, Parasuraman P, Sharma SK, Banerjee T, Karmodiya K, Surolia $\mathrm{N}$, et al. Discovery of a rhodanine class of compounds as inhibitors of plasmodium falciparum enoyl-acyl carrier protein reductase. J Med Chem 2007;50:2665-75.

10. Inamori Y, Okamoto Y, Takegawa Y, Tsujibo H, Sakagami Y, Kumeda $Y$, et al. Insecticidal and antifungal activities of aminorhodanine derivatives. Biosci Biotech Biochem 1998;62:1025-7.

11. Won SJ, Liu CT, Tsao LT, Weng JR, Ko HH, Wang JP, et al. Synthetic chalcones as potential anti-inflammatory and cancer chemopreventive agents. Eur J Med Chem 2005;40:103-12

12. Murugan R, Anbazhagan S, Narayanan SS. Synthesis and in vivo 
antidiabetic activity of novel dispiropyrrolidines through [3+2] cycloaddition reactions with thiazolidinedione and rhodanine derivatives. Eur J Med Chem 2009;44:3272-9.

13. Cutshall NS, O'Day C, Prezhdo M. Rhodanine derivatives as inhibitors of JSP-1. Bioorg Med Chem Lett 2005;15:3374-9.

14. Carter PH, Scherle PA, Muckelbauer JK, Voss ME, Liu RQ, Thompson LA, et al. Photochemically enhanced binding of small molecules to the tumor necrosis factor receptor-1 inhibits the binding of TNF-alpha. Proc Natl Acad Sci U S A 2001;98:11879-84.

15. DegterevA, Lugovskoy A, Cardone M, Mulley B, Wagner G, Mitchison T, et al. Identification of small-molecule inhibitors of interaction between the BH3 domain and bcl-xL. Nat Cell Biol 2001;3:173-82.

16. Helal CJ, Sanner MA, Cooper CB, Gant T, Adam M, Lucas JC, et al.
Discovery and SAR of 2-aminothiazole inhibitors of cyclin-dependent kinase 5/p25 as a potential treatment for alzheimer's disease. Bioorg Med Chem Lett 2004;14:5521-5.

17. Sundaram K, Ravi S. Synthesis, antibacterial activity against MRSA, and in vitro cytotoxic activity against HeLa cell lines of novel 3 - $\alpha$-carboxy ethyl-5-benzylidene rhodanine derivatives. Res Chem Intermed 2015;41:1011-21.

18. Thangamani A. Regiospecific synthesis and biological evaluation of spirooxindolopyrrolizidines via [3+2] cycloaddition of azomethine ylide. Eur J Med Chem 2010;45:6120-6.

19. Mosmann T. Rapid colorimetric assay for cellular growth and survival: Application to proliferation and cytotoxicity assays. J Immunol Methods 1983;65:55-63. 